\title{
How the Market Can Detect Its Own Mispricing - A News Sentiment Index to Detect Irrational Exuberance
}

\author{
Jonas Krinitz \\ University of Freiburg \\ Freiburg, Germany \\ Email: jonas.krinitz@gmail.com
}

\author{
Simon Alfano \\ University of Freiburg \\ Freiburg, Germany \\ simon.alfano@is.uni-freiburg.de
}

\author{
Dirk Neumann \\ University of Freiburg \\ Freiburg, Germany \\ dirk.neumann@is.uni-freiburg.de
}

\begin{abstract}
The emergence of big data analytics enables real time news analysis. Such analysis offers the possibility to instantly extract the sentiment conveyed by any newly published, textual information source. This paper investigates the existence of a causal relationship between news sentiment and stock prices. As such, we apply news sentiment analysis for unstructured, textual data to extract sentiment scores and utilize the Granger-causality test to determine the causal relationship between daily news sentiment scores and the corresponding stock market returns. Upon successfully identifying such a causal relationship with a time lag, we develop a real-time news sentiment index. This news sentiment index serves as a decision-support system in detecting a potential over- or undervaluation of stock prices given the news sentiment of available news sources. Thus, as a novelty, the news sentiment index serves as an early-warning system to detect irrational exuberance.
\end{abstract}

\section{Introduction}

One of the underlying assumptions of economic theory is informational efficiency. Informational efficiency relates to the fact that investors endogenize all available information into their buy- or sell-decisions and thus reflect all available information in market prices. While the classical economic approaches assume perfect informational efficiency as stipulated in the efficient market hypothesis, the field of behavioral economics examines in numerous contributions how and why human behavior in market interactions undermines complete informational efficiency [1]-[3].

In fact, every economist would like to know what will happen tomorrow in the economy. However, despite different behavioral anomalies affecting human decision-making as outlined by behavioral economics research e.g. [4], [5], making robust predictions of future economic developments is a huge challenge both in academic research and financial markets.

Such predictions of future events become less accurate the further we go into the future. In order to develop predictions, economists use knowledge they acquired in the past to detect certain recurring patterns and relationships among the observed variables. This econometric knowledge then effectively translates into extrapolating certain patterns into the future, expecting these historic processes to repeat with a certain probability. In the context of analyzing ever growing data sources of different data variety (e.g. qualitative and quantitative, structured and unstructured data), big data analytics and data science have developed novel approaches to analyze data faster, integrate more varied data sources and run analytics models more accurately, such as machine learning [6], [7].

We have learned that certain events trigger or influence other events, for example the negative causality of inflation and unemployment, or interest rates and exchange rates. Consequently, such insights enable us to understand relationships of relevant economic factors. However, when evaluating empirical data and moving from such abstracted, isolated bilateral relationships to economic behavior observed in markets with a multitude of stakeholders, external and internal influencing factors come into play and contribute to the complexity of economic predictive and forecasting analysis.

To get an insight into the state our economy is in, and to anticipate future events, one approach is to account for government statistics or market data, such as earning reports of stock-listed companies, which typically get published with significant time lags. Hence, predictions on the basis of such data imply a certain time-lag between the point in time to which such data points refer to, and the point in time at which the data is published in order to make a prediction of future events.

One alternative approach is to rely on data with smaller or virtually no time lag. One such approach is to poll the moods and expectations of subjects working in the economy, namely bankers, managers, analysts, brokers, CEOs or companies. Such indices are often published by country, industry or value chain function, such as procurement. Examples include the PMI on either a global scale or, or for specific region (e.g. USA, Japan, UK) and the ifo Index or ZEW in Germany [8]-[10]. Those are updated monthly and well publicized via all different types of German media. It polls the sentiment of firms based in 
the construction industry, manufacturing industry, retail and wholesale business and summarizes their expectations of the current state of the economy and expected development of the next month [11].

However, the ifo Index in its structure rarely surprises us with early insights of emerging events [12]. This weakness of the ifo, is due to its survey based structure. None of the surveyed individuals will share expectations on the economy, which they have not yet translated into actions respectively prices for themselves. The ifo is an index of information that is already priced in. Even with their inside knowledge they themselves are victims of 'irrational exuberance' which makes their survey answers biased. Therefore, we must search an unbiased instrument which allows us to detect emerging 'economic bubbles' and irrational exuberance as early as possible [13].

For that reason, this paper shall focus on finding a new instrument to forecast price developments of a stock market index through the sentiment contained in stockrelevant financial disclosures of the companies listed in a specific index, in order to find an early-warning indicator and develop a new type of sentiment index. This sentiment analysis approach allows us to tap into a new source of sentiment data, allowing us to modulate the sentiment of a business area in real time. To do so, we introduce the literature and ideas on which we base our research in section 2. In section 3 we present the news source, which we analyze in section 4 with text mining to transform it into a usable form and describe how we apply the Grangercausality method. The statistical analysis will be performed in section 5, for the whole data and for sequential subsets. Section 5 will also include the psychological interpretation of our results and the search for 'irrational exuberance'. Section 6 deals with the evaluation of our findings and the development of the new economic index. The advantages of this index and an outlook for future research will be included in the concluding sections 7 and 8 .

\section{Related Work}

In this section we introduce the literature on which we base our analysis and compare the approach we take with related work of this field. Based on the literature and the idea for our research, we will derive our two research questions.

\subsection{Literature on Economic Indices}

The original idea to search for a source of unbiased sentiment derives from Tetlock, Feuerriegel and Hagenau [14][16], who introduced the concept of investigating 'whether news momentum can predict medium-term stock index de- velopment'. Following the idea of Hagenau's paper's title 'Reading All The News at the Same Time', we analyze news to extract sentiment values. The focus in doing so is not based on how a breaking news announcement influences the short-term stock return [17], [18], or to predict the stock markets volatility [19]. Nether do we test noise trade methods, based on sentiment data [20].

Our interest lies in finding a medium-term causal relationship between the stock indices and news sentiments. Similar to the CrisisModeler by Holopainen and Sarlin [21], our objective is to find an instrument, that indicates an emerging crisis. While they build a web-based crisis modeling application with the ability of finding early warning signs, but as a more general modeling solution and for crisis prediction, our interest lies in finding a sentiment instrument to detect the first signs of 'irrational exuberance' in financial markets. A more micro-economic warning mechanism can be found in the work of Betz et al. [22], where the early warning model is not only for an economic region, but specific for banks and their vulnerability to emerging crisis, due to country-specific and bank-specific risks measures. An approach, more similar to our sentiment instrument is utilized by Jansen et al. [23], which studies the influence of consumer confidence on short term stock market development for different countries in the EU. They also use the Granger-causality test in order to find a delayed influence on stock market pricing, but don't search for the specific causal lags but look for a direct and 30 day causality. We will test for more particular significant lag sequences and test if they change over time.

Furthermore, we will include a behavioristic analysis, to determine whether the differences between the sentiment's movement and those of the stock market follow a principle that can be psychologically explained. This analysis will be based on Shiller's search for 'irrational exuberance' and include several cognitive biases to explain different behavior between our time series [13].

\subsection{Research Question}

If we combine the concept of the empirical search for a causal relationship between the market's news sentiment and the CDAX, and the cognitive biases regarding an explanation of investors, reaction to news, we end up with two questions, for which we aim to find the answers.

The research question for this paper can be summed up as:

1) Is there a causal relationship between news sentiment and the German stock market?

2) Can we identify a delay between the estimated news sentiment and the reaction in the market and, how long is it? 


\section{Data}

The source of sentiment data we use are corporate announcements instead of journalistic news. One reason for this is that news about the fundamentals of a company are a better source for predicting earnings and returns of companies [24]. The other purpose is to tap into a trustworthy and non-biased source for new information regarding the economic situation and development of a certain company.

The news announcements we analyze contain information which were not publicly announced before and as a consequence are not yet represented in stock prices. These corporate announcements are of a regulatory nature, meaning reports of the companies status, earning reports, announcements for future plans and ad-hoc publications of voting share developments, all according to the Wertpapierhandelsgesetz $(W p H G)$. This includes annual and half-year financial statements plus additional financial reports regarding the company transparency. We expect the tone of these adhoc news to be more neutral and therefore more likely to represent the real news's meaning.

With this in mind, and given the exclusive use of first-hand news, we do not need to control for exuberant feedback loops, the staggering effect of positive news only triggered by previous positive news, vice versa. All the analyzed news items, we use in this paper, are taken from German stock companies without exclusions, published between January 2004 and July 2011 through the DGAP. The data set consists of 14427 individual news reports on 2013 days in our observation period. Since we are looking for causality between these news and related quantifiable economic figures such as stock index, we will use the Composite DAX (CDAX). The CDAX includes all German stock companies.

\section{Text Mining, Sentiment Analysis and Causality}

This section introduces the methods used to generate our data set and how we are going to analyze it. Text mining is the first step to generate the analyzable sentiment values for every CDAX ad-hoc news announcement. We also take the CDAX for that period. The second step is to introduce the Granger-causality method which we will use to determine if and how the causal relationship between both time series occurs.

\subsection{Sentiment Analysis and Data Set}

Sentiment analysis refers to the analytical method which interprets the meaning of every word through an either positive or negative value. In order to generate these sentiment scores, we need to prepare the text corpus of the news we want to analyze. Therefore, we start by tokenizing the text [25], detecting and inverting negation [26] and removing stop words [27]. Finally, we perform stemming for the remaining text with the Porter stemming algorithm [28] to generate our final text corpus.

In order to create reproducible sentiment scores [29] and due to the very robust results found by Liebmann [30], we use the Net-Optimism metric [31] combined with Henry's Finance-Specific Dictionary [32]. The sentiment scores $S(A)$ in this method are calculated as the difference of positive $W_{\text {pos }}(A)$ and negative $W_{\text {neg }}(A)$ words, divided by the total number of words $W_{\text {tot }}(A)$ in a news announcement.

$$
S(A)=\frac{W_{\text {pos }}(A)-W_{\text {neg }}(A)}{W_{\text {tot }}(A)} \in[-1+1] .
$$

In the next section the sentiment value $S(A)$ for every text corpus of the data set will be used to determine whether we can find a causal relationship between it and the stock market.

\subsection{Causality}

To determine the causal relationship between the sentiment and the CDAX, ((news sentiment $\rightarrow$ CDAX), we will first plot both vectors over time (Figure 1) and scan for an observable dependence or causality. In section 5 we will analyze their relationship empirically.

For Figure 1, we have plotted the de-trended time series of the sentiment values and the CDAX. The sentiment values are the daily aggregated news sentiment which is smoothed with a moving average of 100 . The sentiment values and the CDAX have been standardized to plot them together. If we inspect the two time series we can see a similar course for both, from which we can assume that a causal relationship exists. This assumed causality is not immediate, but occurs after a delay of some length. Also the delay seems to change over time. To confirm this theory and identify how big the delay is, we introduce, as our tool, the Granger-causality method.

\subsection{Granger-Causality-Model}

For the Granger-causality test we use the traditional bivariate approach, as developed by Granger [33]. The basis for the bivariate OLS autoregression is including the particular autoregressive lags $p$ for both vectors

$$
\begin{aligned}
x_{t}= & c_{1}+\alpha_{1} x_{t-1}+\alpha_{2} x_{t-2}+\ldots+\alpha_{p} x_{t-p} \\
& +\beta_{1} y_{t-1}+\beta_{2} y_{t-2}+\ldots+\beta_{p} y_{t-p}+u_{t} .
\end{aligned}
$$

An F-test is built to determine if $y$ causes $x$, based on this regression with 


$$
H_{0}: \beta_{1}=\beta_{2}=\ldots=\beta_{p}=0
$$

meaning $y$ does not Granger-cause $x$ and

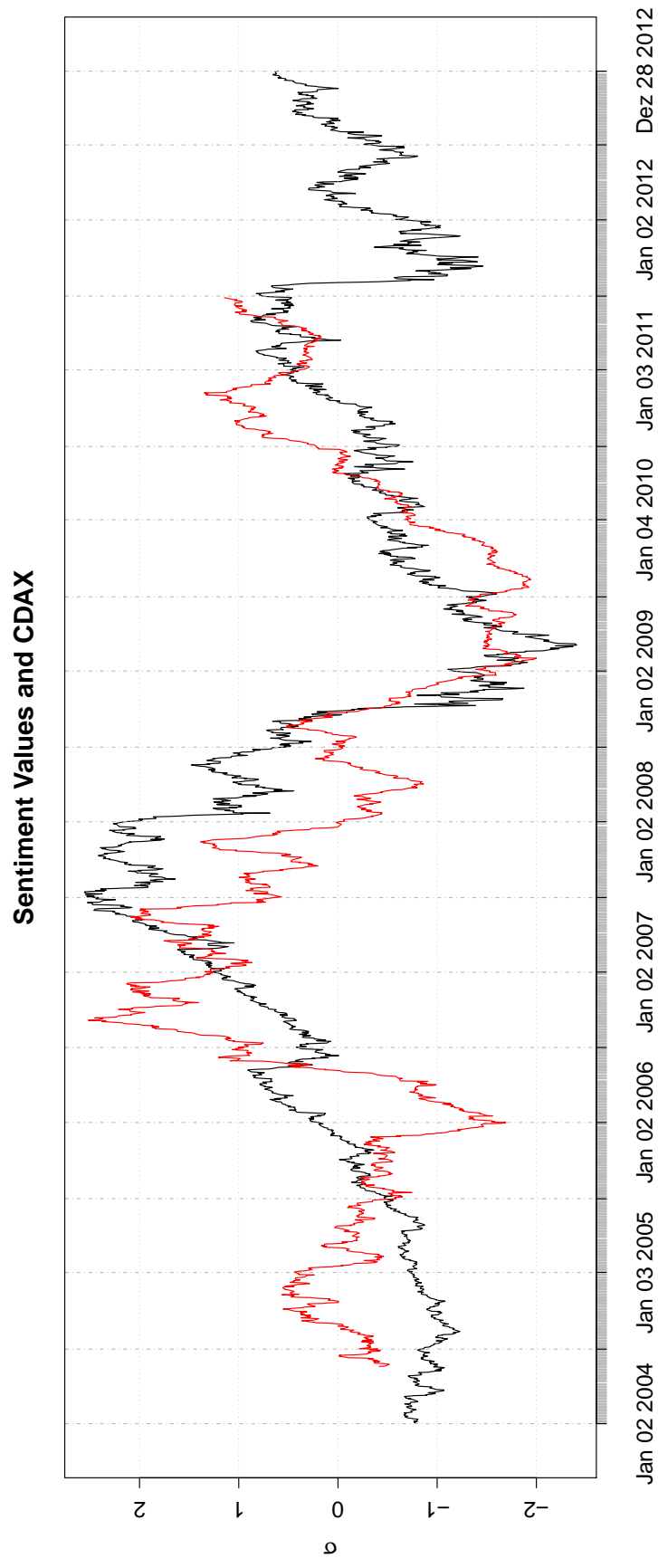

Figure 1. Aggregated, de-trended and Standardized Sentiment Values (red), CDAX (black)

$$
H_{1}:\left\{\beta_{1}, \ldots, \beta_{p}\right\} \neq 0
$$

meaning $y$ does Granger-cause $x$.

The F-test statistic $\left(S_{1}\right)$ will be calculated with the residual sum of squares from equation 2

$$
R S S_{1}=\sum_{t=1}^{T}=\hat{u}_{t}^{2},
$$

and is compared to the RSS of a univariate autoregression for

$$
\begin{array}{r}
x_{t}=c 1+\alpha_{1} x_{t-1}+\ldots+\alpha_{p} x_{t-p}+\epsilon_{t}, \\
R S S_{0}=\sum_{t=1}^{T}=\hat{\epsilon}_{t}^{2} .
\end{array}
$$

The calculate F-statistic

$$
S_{1}=\frac{\left(R S S_{0}-R S S_{1}\right) / p}{R S S_{1} /(T-2 p-1)},
$$

needs to be higher than $1 \%, 5 \%, 10 \%$ critical value for a $F(p, T-2 p-1)$ to reject the $H_{0}$ hypothesis that $y$ do not Granger-cause $x$. The test, even though only asymptotically valid, is enough to determine if a causal relationship exists [33]-[35].

With this model we will work in the next section to determine whether we can find evidence for a causal relationship between the news sentiment and the CDAX.

\section{Applied Granger-Causality}

After discovering signs of causality in Figure 1 we are now applying the Granger-causality method, which was discussed in the last section, with the aim of finding empirical evidence. Until now, we can only assume what kind of lags we have to deal with. However, Granger causality requires an exact lag to calculate the F-statistics and p-values. In order to identify the causal relationship, find the particular lags and prove the hypothesis that the sentiment causes the CDAX, we use a for-loop to repeatedly calculate the multiple Granger-causality test from one lag, up to 360th lags. 


\subsection{Applied Causality to CDAX}

For the application of the Granger-causality test, we have to meet the requirements for OLS models. The time series model we work with is non-stationary, due to the autocorrelation in the CDAX and in sentiment values, which are highly autocorralated for more than 100 lags. To still be able to continue the empirical research we will use a lower moving average of 29 (maximum number of aggregated monthly observations) to level down the autocorrelation of the sentiment series. A further way to deal with nonstationary time series in a bivariate Granger-causality test, is to proceed as [36]-[38] did, and also calculate the Grangercausality for first differences of the de-trended time series. The results for both test-loops, if and for which lag our news sentiment $(y)$ causes CDAX $(x), y \rightarrow x$ are shown in Figure 2

\section{Granger-Causality Test Results}

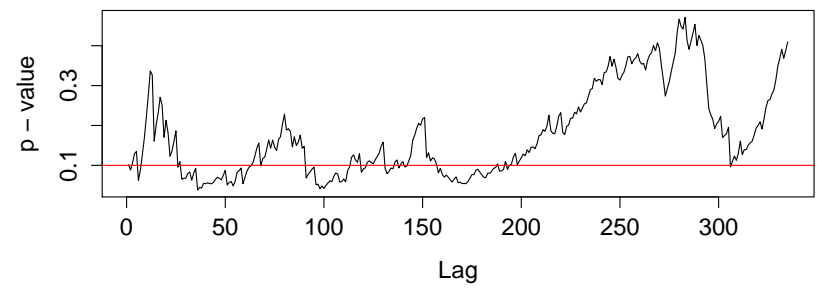

Granger-Causality Test Results of the First Differences

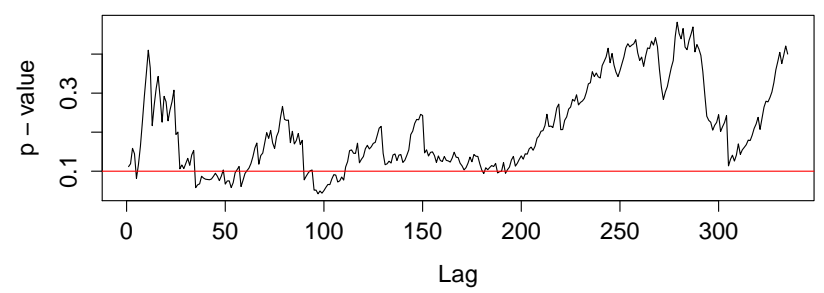

Figure 2. Granger-Causality P-Values for their Respective Lags, for the Standard Time Series and the First Differences

The answer as to whether the sentiment causes a CDAX's reaction and how many days later it reacts on the news sentiment can be answered by the significant $\operatorname{lag}(\mathrm{s})$ in the Granger-causality test. In Figure 2, we can clearly identify several windows of significant lags. The window of significant values for the first differences often lies within those of the standard values. Due to the significance in whole sequences of lags in both models, we can reject $H_{0}$ and say that the causality of $y \rightarrow x$ exists (RQ1) and that its lag is in between one to seven months long. Given the nature of regression, it is always an analysis of means, which is also the case for the Granger-causality. This means that the average causal lag is between 31 and 199 days, and for the first differences between 40 and 115 days. Based on this wide range of significant test results and the visual appearance, with some major- and some minor-appearing delays, we can assume a shift of causal lags through time, with the average lags displayed in Figure 2.

Anticipating the psychological arguments from Shiller [13], Welch and Bikhchandani [39], [40], and the results and conclusion of the test above we have to add a third research question.

3) How does the causal reaction delay change over time?

In order to investigate this hypothesis, we split the time series into four intervals and analyzed the sequences we created. The four intervals reflect a 'pre-crisis' (12. Feb 2004 - 15. Nov 2005), 'boom' (16. Nov 2005 - 27. Aug 2007), 'peak and collapse' (28. Aug 2007 - 09.Jun 2009) and 'post-crisis' (10. Jun 2009 - 27. Jun 2011) periods. This process shrinks our observation subsets to 4 times 447 , due to moving averages and several invalid entries.

For the four new sub-time series, we are also calculating the Granger-causality, just as we did for the whole data. We are taking the non-stationary data, due to its more sensitive appearing p-values. Its $\mathrm{p}$-values and lags are displayed in Table I.

The sequential Granger-causality-test provides enough evidence to support the theory of shifting lags. We can identify four smaller windows of causality within the second and third sequence of the first 90 lags. Based on Figure 1 and 2 we would assume that also higher lags are significant, especially in the first and fourth sequence, but the Granger-causality test only allows us to test for lags $p \approx 100$ and lower, due to the lower number of observations $(n / 4)$ and the regression-based test method, where we use $p * 2$ regressors (see Equation (2)).

Nevertheless, this test confirms the hypothesis of a shifting lag through the period of our observation. In the 'Boom' period and in the 'post-crisis' period, we can measure a low lagged causality, which we weren't able to measure for the period of 'boom' and the 'peak and collapse'.

Overall we have proven: Research Question (RQ) 1 that based on the results displayed in Figure 2 and Table I our news sentiment Granger-causes the CDAX. Furthermore, we have proven the first part of RQ 3 that the lags change over time. Next, we will check if we can find evidence for irrational exuberance that supports the causality we found.

\subsection{Interpretation of Results}

The Granger-causality test has confirmed the hypothesis of lagged and shifting causality, but how can we explain and 
Table I

GRANGER-CAUSALITY P-VALUES FOR THE FouR SPLIT-SEQUENCES. FURTHER NO SIGNIFICANT LAGS UNTIL LAG 90 HAVE BEEN OMITTED.

\begin{tabular}{|c|c|c|c|c|}
\hline \multicolumn{5}{|c|}{ Sequential Granger-Causality-Test Results } \\
\hline Lag & Pre-crisis & Boom & Peak and Collapse & Post-crisis \\
\hline 1 & 0,739186713 & 0,317086723 & 0,173200349 & 0,894068372 \\
\hline 2 & 0,259705621 & 0,404389543 & 0,308727406 & 0,440906866 \\
\hline 3 & 0,203187245 & 0,401557282 & 0,48997493 & 0,66011996 \\
\hline 4 & $0,075723873(*)$ & 0,243863375 & 0,47436175 & 0,74015266 \\
\hline 5 & 0,118608799 & $0,029464127(* *)$ & 0,33678787 & 0,85823339 \\
\hline 6 & 0,162156779 & $0,017391714(* *)$ & $0,05387725(*)$ & 0,92471308 \\
\hline 7 & $0,082115279(*)$ & $0,027118787(* *)$ & $0,05443591(*)$ & 0,859612 \\
\hline 8 & 0,12783948 & $0,037719704(* *)$ & $0,08392403(*)$ & 0,87727711 \\
\hline 9 & 0,23609379 & $0,062486676(*)$ & $0,06847618(*)$ & 0,88999865 \\
\hline 10 & 0,302819936 & $0,096142779(*)$ & $0,04695134(* *)$ & 0,91826532 \\
\hline 11 & 0,387423254 & $0,07834239(*)$ & $0,04880278(* *)$ & 0,7893059 \\
\hline 12 & 0,42135956 & $0,08072422(*)$ & $0,04414824(* *)$ & 0,82886693 \\
\hline 13 & 0,52742917 & 0,101871265 & $0,05506312(*)$ & 0,88989791 \\
\hline 14 & 0,60674901 & 0,167862325 & $0,08357291(*)$ & 0,91744949 \\
\hline 15 & 0,664393483 & 0,136547494 & $0,06068459(*)$ & 0,95520553 \\
\hline 16 & 0,594241502 & 0,186630212 & $0,06407783(*)$ & 0,97813108 \\
\hline 17 & 0,499312711 & 0,234828407 & 0,1093751 & 0,98759885 \\
\hline 18 & 0,463818013 & 0,25812963 & 0,16330707 & 0,99249139 \\
\hline 19 & 0,552530818 & $0,07035775(*)$ & 0,2144849 & 0,94567643 \\
\hline 20 & 0,279041307 & $0,097381613\left(^{*}\right)$ & 0,25263342 & 0,95435524 \\
\hline 21 & 0,477116508 & $0,078661069(*)$ & 0,30920223 & 0,96498079 \\
\hline 22 & 0,409590213 & $0,096366626(*)$ & 0,31625965 & 0,96493442 \\
\hline 23 & 0,486163451 & 0,100905882 & 0,28270996 & 0,94083541 \\
\hline 24 & 0,488800483 & 0,124795073 & 0,31671009 & 0,55406512 \\
\hline 25 & 0,541531178 & 0,161230164 & 0,32648729 & 0,55314947 \\
\hline 26 & 0,34582365 & 0,183414944 & 0,29093068 & 0,57232473 \\
\hline 27 & 0,240793303 & $0,095145385(*)$ & 0,31055511 & 0,58219669 \\
\hline 28 & 0,237790635 & $0,05394625(*)$ & 0,23634992 & 0,64450727 \\
\hline 29 & 0,256062484 & $0,066752148(*)$ & 0,26371494 & 0,56301771 \\
\hline 30 & 0,287604676 & $0,078019286(*)$ & 0,31225518 & 0,6101801 \\
\hline 31 & 0,304621415 & $0,084009667(*)$ & 0,17175076 & 0,63283584 \\
\hline 32 & 0,351037739 & $0,068722687(*)$ & 0,21666136 & 0,66842683 \\
\hline 33 & 0,430488787 & $0,030979123(* *)$ & 0,19001173 & 0,60041813 \\
\hline 34 & 0,323327521 & $0,037391645(* *)$ & 0,20276327 & 0,4491228 \\
\hline 35 & 0,382039819 & $0,047191041(* *)$ & 0,21577365 & 0,51902584 \\
\hline 36 & 0,416538139 & $0,06203028(*)$ & 0,28491049 & 0,59522962 \\
\hline 37 & 0,372597818 & $0,048604594(* *)$ & 0,3457867 & 0,64356775 \\
\hline 38 & 0,338034268 & $0,063456198(*)$ & 0,34249047 & 0,5963476 \\
\hline 39 & 0,396318518 & $0,058405243(*)$ & 0,38061615 & 0,66967157 \\
\hline 40 & 0,495941266 & $0,088867035(*)$ & 0,36105436 & 0,66905946 \\
\hline 41 & 0,542324527 & 0,103970565 & 0,29264415 & 0,61201793 \\
\hline 42 & 0,541478598 & 0,116171941 & 0,288961493 & 0,628036971 \\
\hline 43 & 0,644344267 & 0,140355951 & 0,313112965 & 0,490278682 \\
\hline 44 & 0,671988823 & 0,177297033 & 0,328375827 & 0,301752567 \\
\hline 45 & 0,679684175 & 0,176128172 & 0,381071427 & 0,378249716 \\
\hline 46 & 0,701319475 & 0,168796663 & 0,367731711 & 0,440590805 \\
\hline 47 & 0,756203404 & 0,164090536 & 0,324231073 & 0,440508745 \\
\hline 48 & 0,779422274 & 0,156580075 & 0,386995982 & 0,412768759 \\
\hline 49 & 0,769613133 & 0,152564841 & 0,426050014 & 0,318286558 \\
\hline 50 & 0,817863866 & 0,174490059 & 0,435762243 & 0,256048584 \\
\hline
\end{tabular}

interpret the lag length and changes? For this we will take the cognitive biases which Shiller sees as powerful enough to influence people's behavior away from reason [13].

The shifting of lag length has been proven in Table I. There the significant lags deviated between the four sequences of the time series. If we focus on Figure 1, we can see that the distance between the sentiment and the CDAX grows when the period before has been constantly higher sentiment. When the sentiment reaches the peak and drops afterward, the CDAX continues to grow. This is consistent with Shiller's argumentation. He calls this detachment from fundamentals irrational exuberance. It is a type of herd behavior, triggered by the information cascade, where the additional information of the rational investment decision of a primary investors group reacting to a news leads to a second group reacting. This reaction of the second group has the additional information of the first group already investing, leading to irrational overestimation and thus over-investing. This further detaches market development from the declining news sentiment [39], [40]. Due to the mechanism of the emotional bias it is not likely that this effect happens the other way around.

In this period, emotional bias lets people reject the possible truth of dropping markets, if the result of accepting it would be harmful for them. This can extend and perpetuate herd behavior and the resulting growth periods [41]. The downward correction after the extended period of growth progresses bit by bit, as not all accept the growth inversion immediately. For those investors who still believe the ongoing growth, escalation of commitment lets them commit even more firmly to their investments. This self-perpetuating effect can even accelerate the creation of a bubble. At the point where the majority has to accept the evidence of a 'bubble', the market collapses and adjusts downwards, towards the news sentiment. The causality lag shrinks from a length of up to seven months to around one month.

We can identify this 'irrational exuberance' driven growth in the period before 2008 and again at the end of 2010 for our data.

Based on studied biases in combinations with the observations of repeating and explainable behavior we would expect people, in times of crisis, to be more receptive to good news than in times of growth to bad news. We call this behavior, following Shiller, irrational exuberance.

This knowledge will be used in the next chapter to create an index displaying the found causality.

\section{Sentiment to CDAX Index}

In the previous chapters we have found sufficient evidence to say that the market sentiment $(y)$, which we extracted from corporate news, Granger-causes the stock market, in our case the CDAX $(x)$. The task ahead is now, how to translate the causality into a form, that shows the market sentiment's state and what kind of development the CDAX, or the economy, can expect in the future.

As a trivial index, we could simply take the standardized moving average of 29 or 100 days, sentiment values as shown in Figure 1. Using this approach, the index would assume a more or less average basic sentiment, which only moves above or beyond when good or bad news breaks 
the market and returns if no further news is published. We could simply read the market news sentiment as its own standard deviation. This would be analogous to the ifo growth expectations (Gesch äftserwartungen) [11].

However, the analysis has been based on news from the stock market, and should include the respective stock market in the index. We will construct the index as an over- or underpricing indicator for the related market. To indicate whether the market is overpriced, meaning the stock market (CDAX) would be above the sentiment for a point of time, the index should be negative, due to its expected future return to a lower level, and vice versa. For this concept of an indicator of future development we can take the difference of the standardized 29 days, moving average for the sentiment (SX) and the standardized CDAX (CX),

$$
S T C I=S X-C X
$$

and model them over time, as done in Figure 3.

In doing so, we generate a new time series, which displays the difference between CDAX and sentiment in the differences of standard deviations. To ease the interpretation we added a colored background, functioning as an indicator of a market that is unreasonably detached from the sentiment and its expected future development, with a green upper area and a red area below. In this context red means that the sentiment is lower than the CDAX and green illustrates the opposite. If we assume that the sentiment reflects the market's fundamentals, we can claim that negative values indicate overpricing (red) and positive values indicate underpricing (green). The colors in this context also indicate the expected future development for the CDAX, since we expect it to follow the sentiment after a lag. The area between 1 and 1 is left uncolored, as we always see some minor differences between the two time series, which mark no significant exuberance.

Since this type of index displays the relation of the market sentiment to the stock market itself we are calling this type of index Sentiment to Stock Market Index, short STSMI. In our case for the German stock market, CDAX, the index is now referred to as Sentiment to CDAX Index (STCI).

\section{Advantages and Usability of the Sentiment to Stock Market Index}

In the previous chapters we have analyzed the influence of corporate announcements on the economy. We have translated this causality into a model that illustrates the difference between the news sentiment and economic indices. However, of what use is this model or index compared to already existing ones, such as the PMI, ifo or $Z E W$ indices and statistics issued by the government? The answer lies in the weaknesses all traditional statistics and

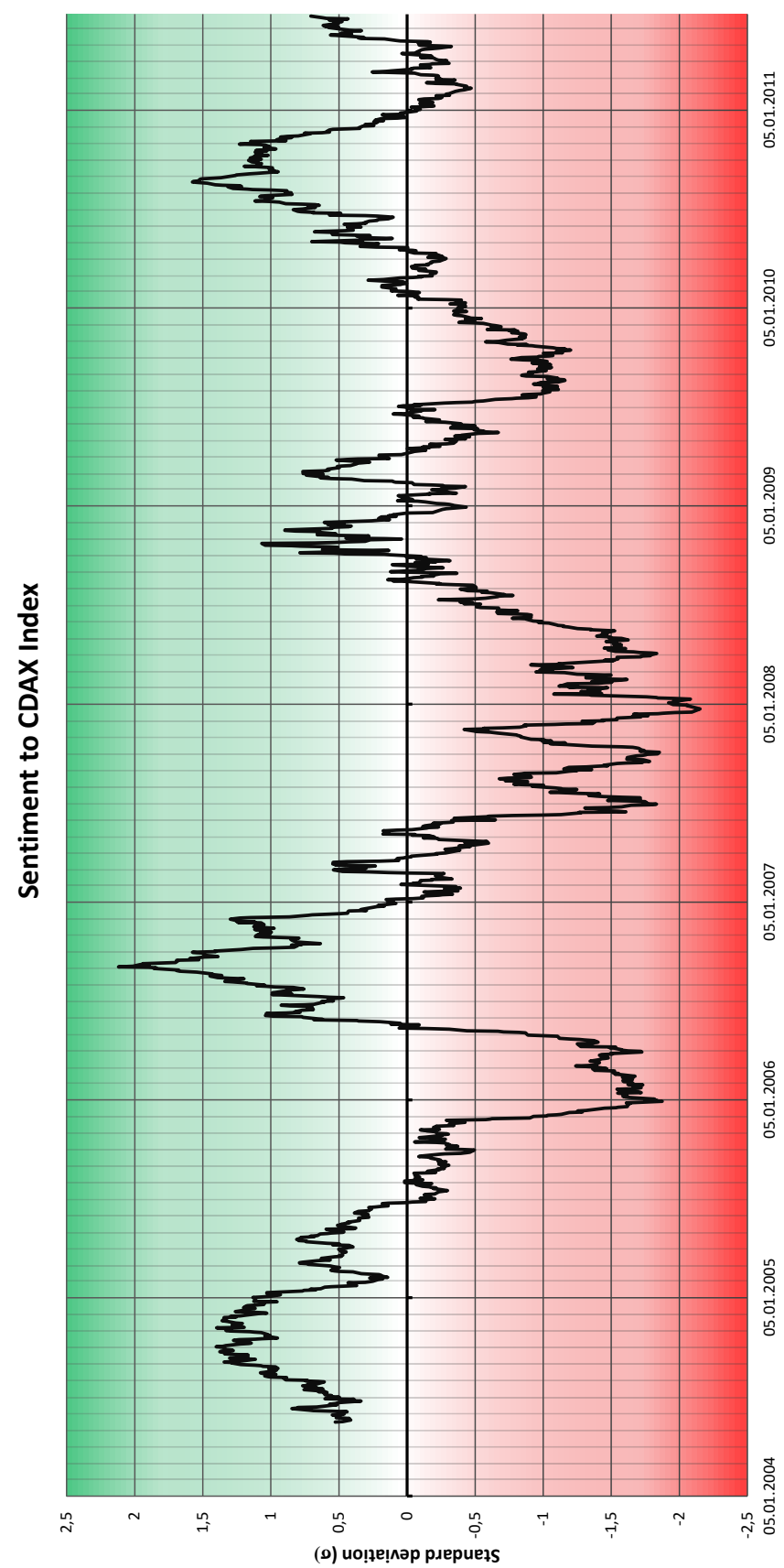

Figure 3. The Sentiment to CDAX Index with a Coloured Background as an Overpricing (red) and Underpricing (green) Indicators

indices have in common.

- Real time analysis of the corporate announcements provides a completely new approach in measuring markets sentiment.

- Low costs, since almost no work is needed to generate and update the index. 
- The analyzed news are of regulatory nature and for all companies obligatory, resulting in an stable and independent news input.

- Less vulnerable to falsifying survey answers, thus unbiased.

- Data is expected to be unbiased and not yet translated into prices.

The indices' core usability is to identify over- or underpricing of aggregated stock markets and detect exaggeration in growth expectations, irrational exuberance [13]. The forecasting potential can be used to identify future trends and emerging 'economic bubbles'. It is not developed for daily trading even though it is updated in real-time. Its forecasting potential is developed for medium-term growth. However, this medium-term growth expectation can change within a period of one day, with every breaking announcement.

\section{Research Outlook}

The basic idea of analyzing market behavior through news sentiment, as done by Hagenau [16] and similarly developed by Tetlock [14] [24] as well as in this paper has been shown to be a reliable indicator for future development. Nevertheless, we have investigated in this paper a time interval which was shaped by devastating bubbles, crises and recessions. To generalize the index further, we should extend the time interval to less volatile times. Furthermore, other markets and sub-markets could be analyzed in a similar fashion to confirm a general causality, transferable to a generalized approach implementing STSMIs globally. It would also be possible to test for different news reception over time, in this and other markets, by using more advanced rolling window Granger-causality tests. Possible research could also include feedback loops and unit root tests, to investigate staggering effects of sentiment on the CDAX. In addition to this it would be interesting to see if our results can be matched by an data-driven sentiment analysis approach.

\section{Conclusion}

In conclusion, the sentiment of corporate announcements has proven to have a strong influence on the development of stock markets, as we proposed in research question 1. We have seen that not only stock markets are news driven, but also that the reception of news is news driven. Long periods of positive news make people over-anticipate the future development of prices. If those prices adjust downwards, the market and also its participants go through more stressful times. The consequence of this phenomenon is that the market's development is closer to that of the news sentiment. This leads to a simple conclusion: happiness (or greed) compels people to turn away from reason. This should not come as a surprise. Nonetheless, the sentiment values reflect a stable relation to the CDAX with psychologically explained lag shifts (RQ 3). These lag shifts, indicate overor underpricing/underestimation of the market's status. The Sentiment to CDAX Index indicates when our assumption of aggregated market value is not reflected in the news we read of those companies we value through market prices. We can not give a definite answer to research question 2 regarding the length of the delay, since the lag is not constant, as explained in the interpretation. Nevertheless, the change in lag length can be expected to follow the rules of 'irrational exuberance' and is, along with the STCI, an indicator for future development and current over- or underestimation of the market.

The approach that Hagenau proposes, and which we adapted, was proven to be a reliable method capturing market sentiment. We have developed it into a non-surveybased sentiment index which compensates for the problems seen in conventional sentiment capturing approaches. It surpasses conventional methods in terms of usability and especially as an early warning signal, still offering a wide psychological and statistical base. The future will show how effective and accurate the STCI is in non-crisis-times and how market participants will adapt to news sentiment analysis.

\section{References}

[1] K. D. Daniel, D. Hirshleifer, and A. Subrahmanyam, "Investor psychology and security market under- and overreactions," The Journal of Finance, vol. 53, no. 6, pp. 1839-1886, 1998.

[2] B. DeLong, A. Shleifer, L. H. Summers, and R. J. Waldmann, "Noise trader risk in financial markets," Journal of Political Economy, vol. 98, no. 4, pp. 703-738, 1990.

[3] R. J. Shiller, "From efficient markets theory to behavioral finance," Journal of Economic Perspectives, vol. 17, no. 1, pp. 83-104, 2003.

[4] D. Kahneman and A. Tversky, "Choices, values, and frames," American Psychologist, vol. 39, no. 4, pp. 341-350, 1984.

[5] A. Tversky and D. Kahneman, "Rational choice and the framing of decisions," The Journal of Business, vol. 59, no. 4 , pp. 251-278, 1986.

[6] IBM, "The four v's of big data," 2013. [Online]. Available: http://www.ibmbigdatahub.com/infographic/four-vs-big-data

[7] H. R. Varian, "Big data: New tricks for econometrics," Journal of Economic Perspectives, vol. 28, no. 2, pp. 3-28, 2014. 
[8] Clemens Fuest, "ifo geschäftsklima deutschland: Ergebnisse des ifo konjunkturtests im mai 2016," 2016.

[9] D. Rehse, "Zew finanzmarktreport: Einschätzungen und erwartungen zu internationalen finanzmarktdaten," 2016. [Online]. Available: zew.eu

[10] C. Williamson, "Markit global sector pmi: Technology and financials prop up global growth in may," 2016. [Online]. Available: https://www.markiteconomics.com/

[11] Hans-Werner Sinn, "Ergebnisse des ifo konjunkturtests im oktober 2015," 2015. [Online]. Available: http://www.cesifogroup.de/de/ifoHome/facts/Survey-Results/BusinessClimate/Geschaeftsklima-Archiv/2015/Geschaeftsklima20151026.html

[12] R. Lehmann, W.-D. Speich, R. Straube, and G. Vogt, "Funktioniert der ifo konjunkturtest auch in wirtschaftlichen krisenzeiten? eine analyse der zusammenhänge zwischen ifo geschäftsklima und amtlichen konjunkturdaten für sachsen," Dresden, 2010.

[13] R. J. Shiller, Irrational Exuberance, revised and expanded third edition ed. Princeton: Princeton University Press, 2015.

[14] P. C. Tetlock, "Giving content to investor sentiment: The role of media in the stock market," The Journal of Finance, vol. LXII, no. 3, 2007.

[15] S. Feuerriegel, S. F. Heitzmann, and D. Neumann, "Do investors read too much into news? how news sentiment causes price formation," in 2015 48th Hawaii International Conference on System Sciences (HICSS), pp. 4803-4812.

[16] M. Hagenau, M. Hauser, M. Liebmann, and D. Neumann, "Reading all the news at the same time: Predicting mid-term stock price developments based on news momentum," 46th Hawaii International Conference on System Sciences, 2013.

[17] R. P. Schumaker and H. Chen, "Textual analysis of stock market prediction using breaking financial news: The azfintext system," ACM Transaktions on Information Systems, vol. 27(2), pp. 1-19, 2009.

[18] M.-A. Mittermayer, "Forecasting intraday stock price trends with text mining techniques," in 37th Annual Hawaii International Conference on System Sciences, 2004. Proceedings of the, p. $10 \mathrm{pp}$.

[19] Y.-H. Wang, A. Keswani, and S. J. Taylor, "The relationships between sentiment, returns and volatility," International Journal of Forecasting, vol. 22, 2006.

[20] M. W. Uhl, "Reuters sentiment and stock returns," THE JOURNAL OF BEHAVIORAL FINANCE, vol. 15, 2014.

[21] M. Holopainen and P. Sarlin, "Crisismodeler: A tool for exploring crisis predictions," 2015 IEEE Symposium Series on Computational Intelligence, 2015.

[22] F. Betz, S. Oprica, T. A. Peltonen, and P. Sarlin, "Predicting distress in european banks," International Journal of Forecasting, vol. 20, 2014.
[23] W. Jos Jansen and N. J. Nahuis, "The stock market and consumer confidence: European evidence," Economics Letters, vol. $79,2003$.

[24] P. C. Tetlock, M. Saar-Tsechansky, and S. Macskassy, "More than words: Quantifying language to measure firms' fundamentals," The Journal of Finance, vol. LXIII, no. 3, 2008.

[25] C. D. Manning and H. Schütze, Foundations of Statistical Natural Language Processing. Cambridge, MA: MIT Press, 1999.

[26] N. Pröllochs, S. Feuerriegel, and D. Neumann, "Enhancing sentiment analysis of financial news by detecting negation scopes," in 48th Hawaii International Conference on System Sciences (HICSS). IEEE Computer Society, 2015.

[27] D. Lewis, Y. Yang, T. Rose, and F. Li, "Rcv1: A new benchmark collection for text categorization research," Journal of Machine Learning Research, vol. 5, pp. 361-397, 2004.

[28] M. F. Porter, "An algorithm for suffix stripping," Program, vol. 14 , no. 3 , pp. 130-137, 1980.

[29] T. Loughran and B. McDonald, "Textual analysis in accounting and finance: A survey," Journal of Accounting Research, vol. 54, no. 4, pp. 1187-1230, 2016

[30] M. Liebmann, M. Hagenau, M. Hussler, and D. Neumann, "Predicting analyst and investor interpretation measuring subjective tone in financial news," working paper.

[31] E. A. Demers and C. Vega, "Soft information in earnings announcements: News or noise? insead working paper no. 2010/33/ac," SSRN Electronic Journal, 2010.

[32] E. Henry, "Are investors influenced by how earnings press releases are written?" Journal of Business Communication, vol. 45, no. 4, pp. 363-407, 2008.

[33] C. W. J. Granger, "Investigating causal relations by econometric models and cross-spectral methods," Econometrica, vol. 37, no. 3, p. 424, 1969.

[34] J. D. Hamilton, Time Series Analysis. Princeton, N.J. Princeton University Press, 1994.

[35] Z. Bai, W.-K. Wong, and B. Zhang, "Multivariate linear and nonlinear causality tests," Mathematics and Computers in Simulation, vol. 81, no. 1, pp. 5-17, 2010.

[36] L. J. Christiano and L. Ljungqvist, "Money does grangercause output in the bivariate money-output relation," Journal Of Monetary Economics, vol. 22, no. 2, pp. 217-235, 1988.

[37] J. H. Stock and M. W. Watson, "Interpreting the evidence on money-money-income causality," Journal of Econometrics, vol. 40, no. 1, pp. 161-181, 1989.

[38] E. L. Feige and D. K. Pearce, "The causal causal relationship between money and income: Some caveats for time series analysis," The Review of Economics and Statistics, vol. 61, no. 4, pp. 521-533, 1979.

[39] I. Welch, "Sequential sales, learning, and cascades," The Journal of Finance, vol. 47, no. 2, pp. 695-732, 1992. 
[40] S. Bikhchandani, D. Hirshleifer, and I. Welch, "A theory of fads, fashion, custom, and cultural change as informational cascades," Journal of Political Economy, vol. 100, no. 5, p. 992, 1992.

[41] F. Wieber, J. L. Thurmer, and P. M. Gollwitzer, "Attenuating the escalation of commitment to a faltering project in decision-making groups: An implementation intention approach," Social Psychological and Personality Science, vol. 6, no. 5, pp. 587-595, 2015. 